\title{
EXPLICANDO LA CONMUTACION LABORAL: EL CASO DE LA PATAGONIA CHILENA
}

\section{EXPLAINING LABOR COMMUTATION: \\ THE CASE OF CHILEAN PATAGONIA}

\section{LUZ MARIA FERRADA}

Universidad de Los Lagos

\section{SERGIO SOZA-AMIGO}

Universidad Austral de Chile

\section{CLAUDIO MANCILLA}

Universidad de Los Lagos

\begin{abstract}
This article evaluates labor commutation from different territories of Chilean Patagonia towards its Nodal Centers: Valdivia, Osorno, Puerto Montt, Coyhaique and Punta Arenas. Commutation is verified in low qualified jobs, with cost of transport and distance being clear determinants; furthermore, the greater population size of the Centers is a pull factor when distances are smaller. At the same time, although some common patterns are obtained between Valdivia, Osorno and Puerto Montt, others have a distinct impact. Finally, all these factors allow us to outline territorially differentiated proposals for public policies.
\end{abstract}

Keywords: Commutation, labor mobility, regional labor market.

JEL Classification: R23, J62, J69.

\section{Resumen}

En este artículo se evalúa la conmutación laboral desde distintos territorios de la Patagonia chilena hacia sus Centros Nodales: Valdivia, Osorno, Puerto 
Montt, Coyhaique y Punta Arenas. Se verifica conmutación en empleos de baja cualificación. Son determinantes el costo de transporte y las distancias medias; además, el mayor tamaño de población de los Centros es un factor de atracción cuando las distancias son menores. A su vez, si bien se obtienen patrones comunes entre Valdivia, Osorno y Puerto Montt, hay otros que ejercen impacto distinto; todo ello permite finalmente esbozar propuestas de políticas públicas diferenciadas territorialmente.

Palabras clave: Conmutación, movilidad laboral, mercado del trabajo regional.

Clasificación JEL: R23, J62, J69.

\section{INTRODUCCION}

Este trabajo tiene como área de estudio al espacio geográfico localizado entre las regiones chilenas de Los Ríos y Magallanes (anexo). De acuerdo con la descripción de Said (2012), esta corresponde al territorio imaginario denominado Patagonia occidental, y en este caso Patagonia chilena. Se trata de un territorio que ha concitado un particular interés por los investigadores desde tiempos remotos, posiblemente por su localización espacial y diversidad geográfica, entre otras características.

No obstante, se ha observando que existen ciertas diferencias entre las que llamamos regiones de la Patagonia sur, donde se localizan las regiones de Aysén y Magallanes, y Patagonia norte, que comprenden a las regiones de Los Ríos y Los Lagos (Ferrada, Soza-Amigo y Mancilla, 2017).

En el sur existe un mayor nivel de accidentes geográficos y distancias entre comunas, en consecuencia, muchos de sus habitantes residen en localidades muy apartadas y cuando deciden trabajar en otro lugar deben asumir costos y tiempos de desplazamiento elevados. Al contrario, los habitantes de la Patagonia norte recorren menores distancias y asumen menos costos de transporte y, por tanto, en este caso trasladarse implica exigir un menor salario de reserva; lo anterior permite una mayor movilidad, logrando de esta forma mejores oportunidades de empleo. A su vez, en la Patagonia norte históricamente han existido mayores elementos que relacionan a los habitantes del territorio, por ejemplo aspectos culturales como pueblos originarios y colonizadores (Said, 2012), y antecedentes económicos como la matriz productiva (Soza-Amigo, 2016).

La literatura ha señalado a la conmutación como un comportamiento moderno, cada vez más frecuente, posiblemente explicado por la disminución del costo de traslado derivado de los avances tecnológicos asociados al mercado del transporte (Abalos y Paredes, 2016). Siguiendo este razonamiento, la distancia debiese ser un 
factor con bajo impacto en la decisión de trabajar en un lugar distinto al de residencia. Sin embargo, la situación puede ser muy distinta en los territorios estudiados. Los costos de transporte están asociados a la distancia y a la disponibilidad de estos medios. En este trabajo nos proponemos dar algunas respuestas en esta dirección, explicando los movimientos espaciales con motivos laborales al interior de la Patagonia chilena.

En este espacio geográfico se observa cierto grado de concentración poblacional (INE, 2016) en 5 comunas: Valdivia, Puerto Montt, Osorno, Coyhaique y Punta Arenas, que denominamos Centros Nodales (Soza-Amigo, Rosales y Aroca, 2016). Estas áreas espaciales presentan algunas ventajas de localización, por lo que podrían ejercer ciertas fuerzas centrípetas (Fujita y Krugman, 2004) derivadas de economías de aglomeración y de urbanización que en ellas ocurren, lo que hace que tengan mayor actividad económica, y consecuentemente una mayor demanda relativa de trabajos. En consecuencia, son lugares que pueden especializarse en producción, resultando atractivas para trabajar, como lo señala Aroca y Atienza (2008) para el caso de la región de Antofagasta; al contrario, otras localidades pudiesen transformarse en centros de consumo potenciando nuevas formas de desarrollo, como se observa en el caso de la comuna de Puerto Varas de la región de Los Lagos.

A su vez, existen factores como las características sociodemográficas y aspectos del mercado laboral de los territorios que pueden afectar a la conmutación, es así como Jamett y Paredes (2013) prueban su impacto en la conmutación de larga distancia en Chile. Así, características del mercado del trabajo como el tamaño de la población económicamente activa, la demanda laboral, las posibilidades de salario y las condiciones laborales, entre otras, pudiesen influir en la decisión de trabajar en un territorio distinto del lugar de residencia. Además, como Asencio y Pino (2015) han verificado para la región del Biobío, la conmutación pudiese estar explicada también por factores asociados al género. En este trabajo se analizan estas variables, sin embargo, nos plantemos que es muy posible que existan ciertos factores que expliquen la conmutación a los Centros Nodales y que sean comunes entre ellos, por ejemplo algunos elementos de la estructura productiva o ciertas características socioculturales. En este sentido, se verificará si las variables explicativas de la conmutación tienen igual o distinto impacto en explicar los flujos de conmutación hacia los Centros Nodales del norte y sur de la Patagonia. Esto es particularmente novedoso, pues no se han realizado estudios de este tipo en la zona, lo que permitirá esbozar algunas propuestas de políticas públicas diferenciadas espacialmente.

Así, los objetivos a desarrollar son: (1) estimar el impacto de la distancia, los costos de transporte, la población y otros factores respecto de las decisiones de movilidad desde distintos territorios de la Patagonia chilena hacia sus Centros Nodales; (2) evaluar si existen otros factores que sean comunes para explicar la conmutación intra-Patagonia hacia los distintos Centros Nodales de ella; y (3) constatar si existen 
diferencias significativas en el efecto de las variables que explican la conmutación entre Centros Nodales.

Con todo, en el estudio nos proponemos demostrar 4 hipótesis:

- $H_{01}$ : La Patagonia tiene un comportamiento moderno en el sentido que el costo de traslado y la distancia entre territorios de origen (residencia) y los Centros Nodales (lugar de trabajo) no impacta significativamente a la conmutación hacia ellos.

- $H_{02}$ : Las características laborales de los territorios de residencia no impactan en conmutar hacia los Centros Nodales de la Patagonia chilena.

- $H_{03}$ : La conmutación con motivos laborales desde distintos territorios de la Patagonia hacia los Centro Nodales está también impactada por factores comunes a toda la zona.

- $H_{04}$ : Existen diferencias significativas en el efecto de las variables conocidas para explicar la conmutación hacia los distintos Centros Nodales de la Patagonia.

En los siguientes apartados se explica la matriz de datos, la metodología utilizada, para posteriormente presentar los resultados y, finalmente, concluir respondiendo a cada una de las hipótesis, con base en las estimaciones realizadas.

\section{DATOS}

Para abordar esta investigación se ha construido una base de datos con los antecedentes de conmutación que provienen de la Encuesta Nacional de Empleo (ENE) en los trimestres móviles octubre a diciembre del 2010 al 2015. Esta encuesta tiene representatividad estadística a nivel regional, provincial y en las ciudades localizadas en las comunas consideradas aquí como Centros Nodales. Sin embargo, el número de observaciones en la mayoría de las comunas de la Patagonia es muy pequeño, sin representatividad estadística. Por tanto se utiliza la estrategia de agrupar las comunas de acuerdo con la cercanía e información disponible, construyendo así lo que denominamos territorios de residencia, así como se indica en el Tabla 1.

Luego se calcula la movilidad desde distintos territorios patagónicos hacia sus Centros Nodales. Así por ejemplo, la conmutación laboral hacia el Centro Nodal de Valdivia puede provenir de personas que residen en diferentes territorios de la Patagonia, 8 en total. Lo mismo sucede con los Centros de Coyhaique y Punta Arenas; y en el caso de Osorno y Puerto Montt, se calcula el número de trabajadores que provienen de 10 territorios de residencia en cada caso. Con todo, considerando que el análisis es para 6 años, se obtiene un panel de 264 observaciones en total. 


\section{TABLA 1}

\section{DEFINICION DE TERRITORIOS DE RESIDENCIA POR NODO}

\begin{tabular}{|c|c|c|c|c|c|}
\hline & \multicolumn{5}{|c|}{ Centro Nodal (Destino) } \\
\hline & Valdivia & Osorno & P. Montt & Coyhaique & P. Arenas \\
\hline $\begin{array}{l}\text { Territorios } \\
\text { de residencia } \\
\text { (origen) }\end{array}$ & $\begin{array}{l}\text { Osorno; } \\
\text { Puerto Montt; } \\
\text { Coyhaique; } \\
\text { Punta Arenas; } \\
\text { resto región } \\
\text { Los Ríos; } \\
\text { resto región } \\
\text { Los Lagos; } \\
\text { resto región } \\
\text { Aysén; } \\
\text { resto región } \\
\text { Magallanes. }\end{array}$ & $\begin{array}{l}\text { Valdivia; } \\
\text { Puerto Montt; } \\
\text { Coyhaique; } \\
\text { Punta Arenas; } \\
\text { resto región } \\
\text { Los Ríos; } \\
\text { comunas de } \\
\text { provincia } \\
\text { de Osorno } \\
\text { (excluyendo } \\
\text { la comuna } \\
\text { de Osorno); } \\
\text { comunas } \\
\text { de la } \\
\text { provincia de } \\
\text { Llanquihue; } \\
\text { resto región } \\
\text { Los Lagos; } \\
\text { resto región } \\
\text { Aysén; } \\
\text { resto región } \\
\text { Magallanes }\end{array}$ & $\begin{array}{l}\text { Valdivia; } \\
\text { Osorno; } \\
\text { Coyhaique; } \\
\text { Punta Arenas; } \\
\text { comunas de } \\
\text { Maullín y } \\
\text { Fresia; otras } \\
\text { comunas } \\
\text { de la } \\
\text { provincia de } \\
\text { Llanquihue; } \\
\text { resto región } \\
\text { Los Ríos; } \\
\text { resto región } \\
\text { Los Lagos; } \\
\text { resto región } \\
\text { Aysén; } \\
\text { resto región } \\
\text { Magallanes }\end{array}$ & $\begin{array}{l}\text { Valdivia; } \\
\text { Osorno; } \\
\text { Puerto Montt; } \\
\text { Punta Arenas; } \\
\text { resto región } \\
\text { Los Ríos; } \\
\text { resto región } \\
\text { Los Lagos; } \\
\text { resto región } \\
\text { Aysén; } \\
\text { resto región } \\
\text { Magallanes }\end{array}$ & $\begin{array}{l}\text { Valdivia; } \\
\text { Osorno; } \\
\text { Puerto } \\
\text { Montt; } \\
\text { Coyhaique; } \\
\text { resto región } \\
\text { Los Ríos; } \\
\text { resto región } \\
\text { Los Lagos; } \\
\text { resto región } \\
\text { Aysén; } \\
\text { resto región } \\
\text { Magallanes }\end{array}$ \\
\hline $\begin{array}{l}\text { Número de } \\
\text { territorios }\end{array}$ & 8 & 10 & 10 & 8 & 8 \\
\hline $\begin{array}{l}\text { Número } \\
\text { observaciones }\end{array}$ & 48 & 60 & 60 & 48 & 48 \\
\hline
\end{tabular}

Fuente: Elaboración propia.

De este modo, la variable endógena es el número de trabajadores que conmutan desde un territorio a un Centro Nodal, en relación con el número total de trabajadores de la Patagonia que conmutan a ese Centro Nodal, en un período determinado expresado en logaritmo natural. Luego, la información se ordena como se indica en la Figura 1.

Así, el vector de variables endógenas de la matriz y corresponde a la proporción de conmutantes en el trimestre del año $t$, desde el territorio de origen (o residencia) $i$, a cada uno de los 5 Centros Nodales $j$ (destino).

A su vez, $X$ representa el vector de $k$ variables explicativas asociadas que se definen a partir de un análisis correlacional entre distintos factores y la conmutación (Ferrada, Soza-Amigo y Mancilla, 2017), las que son incluidas según se describe en la Tabla 2. 


\section{FIGURA 1}

\section{MATRIZ DE DATOS}

\begin{tabular}{|c|c|c|c|c|}
\hline \multirow{2}{*}{ Territorio destino } & \multirow{2}{*}{ Año } & \multirow{2}{*}{ Territorio origen } & \multicolumn{2}{|c|}{ Variables } \\
\hline & & & $Y$ & $X$ \\
\hline \multirow{7}{*}{$j=1$} & \multirow{3}{*}{$t=1$} & $i=1$ & \multirow{3}{*}{$Y_{j t i}$} & \multirow{3}{*}{$X_{j t i}$} \\
\hline & &.. & & \\
\hline & & $i=60$ & & \\
\hline & . & & & . \\
\hline & \multirow{3}{*}{$t=6$} & $i=1$ & \multirow{3}{*}{$Y_{j t i}$} & \multirow{3}{*}{$X_{j t i}$} \\
\hline & & . & & \\
\hline & & $i=60$ & & \\
\hline . &. & . & . & . \\
\hline \multirow{8}{*}{$j=5$} & \multirow{3}{*}{$t=1$} & $i=1$ & \multirow{3}{*}{$Y_{j t i}$} & \multirow{3}{*}{$X_{j t i}$} \\
\hline & & . & & \\
\hline & & $i=60$ & & \\
\hline & . & & & . \\
\hline & . & & & \\
\hline & \multirow{3}{*}{$t=6$} & $i=1$ & \multirow{3}{*}{$Y_{j t i}$} & \multirow{3}{*}{$X_{j t i}$} \\
\hline & & . & & \\
\hline & & $i=60$ & & \\
\hline
\end{tabular}

Fuente: Elaboración propia.

Como ya se ha indicado, la principal fuente de información corresponde a la ENE 2010 al 2015, de ellas se obtienen los datos de la variable endógena, los antecedentes para construir los indicadores de inactividad, fuerza de trabajo femenina, educación, inactividad y edad, señalados en la Tabla 2. El ingreso monetario se obtiene de las encuestas Caracterización Socioeconómica Nacional (CASEN) disponibles para 2011,2013 y 2015. 
En cuanto a la distancia, esta se estima como el promedio de kilómetros entre las comunas de residencia, perteneciente a un territorio de un Centro Nodal (destino); por ejemplo, cuando analizamos la conmutación al Centro Nodal Osorno, un territorio de residencia son las comunas de la provincia de Osorno (excluyendo la comuna de Osorno), por tanto, la distancia aquí calculada corresponde al promedio de kilómetros que una persona debiese recorrer desde cada comuna hacia el Centro de comuna de Osorno. Los datos de distancia provienen del sitio web del Ministerio de Obras Públicas de Chile.

\section{TABLA 2}

\section{DEFINICION DE VARIABLES}

\begin{tabular}{|c|c|c|c|}
\hline Variable & Definición & Media & Desv. Típica \\
\hline Conmuta & Porcentaje de trabajadores que conmutan & 0,33 & 0,88 \\
\hline FTrabajo_mujer & $\begin{array}{l}\text { Proporción de fuerza de trabajo que es } \\
\text { femenina }\end{array}$ & 0,2362 & 0,0351 \\
\hline Educa_superior & $\begin{array}{l}\text { Número de educados con nivel superior, } \\
\text { dividido por el número total de trabajadores } \\
\text { en el territorio }\end{array}$ & 0,2017 & 0,0810 \\
\hline Inactividad & $\begin{array}{l}\text { Número de personas inactivas en proporción } \\
\text { al número de personas de } 15 \text { años y más en } \\
\text { el territorio }\end{array}$ & 0,3784 & 0,0522 \\
\hline Edad3 & $\begin{array}{l}\text { Proporción de fuerza de trabajo que tiene } \\
45 \text { o más años de edad }\end{array}$ & 0,4851 & 0,0478 \\
\hline lng_monetario & $\begin{array}{l}\text { Ingreso monetario promedio obtenido por } \\
\text { los hogares del territorio de residencia en } \\
\$ \text { de } 2015\end{array}$ & 254.171 & 70.466 \\
\hline Distancia & $\begin{array}{l}\text { Cantidad de kilómetros promedio entre las } \\
\text { distintas zonas y el Centro Nodal, según la } \\
\text { ruta caminera más cercana }\end{array}$ & 927,08 & 812,68 \\
\hline Costo & $\begin{array}{l}\text { Costos medios en pesos chilenos de traslado } \\
\text { entre los territorios de residencia a los Centros } \\
\text { Nodales, en el medio de transporte más usado }\end{array}$ & 36.494 & 30.093 \\
\hline Calidad_empleo & Indicador de calidad de empleo en el territorio & 0,7985 & 0,0699 \\
\hline Ipoblacion & Índice de población & 14,148 & 20,381 \\
\hline$I_{p o b} *$ dist $^{-1}$ & $\begin{array}{l}\text { Índice de población relativizado por la } \\
\text { distancia }\end{array}$ & 0,0592 & 0,1181 \\
\hline
\end{tabular}

Fuente: Elaboración propia. 
Los costos de traslado se obtienen por medio de consultas telefónicas, y corresponden al promedio del valor monetario (expresados en pesos de 2016) entre cada una de las comunas pertenecientes a la zona de residencia y un Centro Nodal; en ese sentido, se asume que el costo relativo de los pasajes se ha mantenido en el tiempo de análisis.

Como se observa en la Tabla 2, adicionalmente se calculan tres índices: de calidad de empleo, de población y de población relativo a la distancia, lo que se explica a continuación.

- Indicador de calidad de empleo: señala las condiciones laborales promedio en la zona de residencia considerando 4 aspectos: condiciones contractuales, seguridad social, derechos laborales. Se estima como un promedio simple entre las variables la proporción de trabajadores con derecho a vacaciones, con contrato laboral, con seguro de salud y con seguridad social. Los datos provienen de la ENE.

- El indicador de población: se trata de tener una medida que indique la fuerza de atracción que ejerce un Centro Nodal con relación al resto del territorio por el hecho de tener una concentración relativa de población. Con base en las estimaciones de población del INE se calcula como:

$$
\text { Ipoblacion }=\frac{\text { Población del Centro Nodal de destino }}{\text { Promedio de población comunal del territorio de residencia }}
$$

De esta forma se esperaría que mientras mayor sea el indicador, mayor es el peso de la concentración de población, lo que atraería a más conmutantes laborales hacia el Centro Nodal.

- Indicador combinado entre población y distancia: la idea es observar cómo impacta en la conmutación el peso de la concentración urbana dependiendo de la distancia, para ello se estima un indicador como:

$$
I p o{ }^{*} \text { dist }^{-1}=\left(\frac{\text { Población del Centro Nodal de destino }}{\text { Promedio de población comunal del territorio de residencia }}\right) * \text { Distancia al } \mathrm{CN}^{-1}
$$

En este sentido se espera que el factor de atracción que produce la población sea menor en territorios más distantes.

\section{METODOLOGIA}

Para desarrollar cada objetivo específico (OE) se define una metodología de estimación, su aplicación permitirá luego verificar cada una de las hipótesis planteadas. A continuación se describen. 
OE1: Estimar el impacto de la distancia, los costos de transporte, la población y otros factores sobre las decisiones de movilidad desde distintos territorios de la Patagonia chilena hacia sus Centros Nodales

El procedimiento en este caso es mediante una estimación por mínimos cuadrados generalizados (MCG) para todas las observaciones, como se indica en las siguientes ecuaciones:

$$
\begin{gathered}
\text { Inconmuta }_{i t}=\beta x_{i j t}+\mu_{t j i} \\
\text { lnconmuta }_{i t}=\beta x_{i j t}+\varphi D_{j}+\mu_{t j i}
\end{gathered}
$$

De esta forma, en ambas ecuaciones la variable endógena se expresa en logaritmo natural y la matriz de variables $\mathrm{X}$ representa las características asociadas a antecedentes físicos (distancia, costo, población), del trabajo (inactivos, fuerza de trabajo de la mujer, calidad de empleo) y, de la población (edad, ingreso monetario). En la ecuación 2 se agregan variables dummy, $D$, que indican el Centro Nodal al que se desplaza la proporción de trabajadores que conmutan, desde el territorio $i$ al Centro $j$ en el trimestre octubre-diciembre del año $t$. A su vez, $\mu$ representa el término de perturbación con distribución normal, media cero y varianza constante. En estas condiciones se realizará la inferencia, donde $\beta$ y $\varphi$ son los parámetros estimados que señalan el impacto de las variables explicativas en la posibilidad de conmutar a un Centro Nodal de la Patagonia chilena. Por tanto, mediante esta modelación se verificarán las hipótesis 1 y 2.

OE2: Evaluar si existen otros factores que sean comunes para explicar la conmutación intra-Patagonia hacia los distintos Centros Nodales de ella

La decisión de trabajar en un lugar distinto al lugar de residencia depende de las variables incluidas en la ecuación 1 o 2, pero también de otras, y algunas de ellas posiblemente sean comunes a los distintos territorios; en estas circunstancias, si se intenta explicar la movilidad laboral mediante una única estimación, aunque se conozcan dichos factores, como son iguales entre los territorios, no pueden incluirse en la ecuación. En este caso, dicho efecto queda recogido en las perturbaciones de la estimación.

La propuesta metodológica para verificar si existen factores comunes consiste en estimar una ecuación por Centro Nodal, calculando 5 regresiones mediante MCG (como en la ecuación 1), para luego obtener los residuos de cada estimación, y finalmente, probar si hay correlación entre los distintos vectores de error, verificando la hipótesis nula de ausencia de correlación entre perturbaciones de distintas ecuaciones.

Para lo anterior se estima la matriz de correlación de Pearson entre los residuos de las ecuaciones señaladas. Sin embargo, es posible que los errores de algunas 
ecuaciones, no necesariamente todas, estén asociados. En consecuencia, la idea es también evaluar si hay correlación entre un grupo de vectores residuales, para ello se utiliza el test de multiplicadores de Lagrange (Breusch y Pagan, 1980), a partir del contraste (Greene, 1999: 588), así como se señala en la ecuación 3.

$$
\lambda_{L M}=N \sum_{m=2}^{M} \sum_{j=1}^{n-1} r_{m j}^{2} \approx \frac{\chi_{M(M-1)}^{2}}{2}
$$

Siendo $N$ el número de observaciones, $M$ el número de variables endógenas, $r_{m j}$ el estimador del coeficiente de correlación lineal entre las perturbaciones de dos zonas: $r_{i j}=\frac{\hat{\sigma}_{m j}}{\sqrt{\hat{\sigma}_{m m} \hat{\sigma}_{j j}}}$. A causa de que en esta investigación se tienen distintos números de observaciones en las ecuaciones, el estimador consistente que se utiliza para $\sigma_{m j}$ es $\hat{\sigma}_{m j}=\frac{e_{m}^{\prime} e_{j}}{\max \left(N_{m}, N_{j}\right)}$; esto es, para estimar el coeficiente de correlación se utiliza el máximo de los dos tamaños muestrales correspondientes a las dos zonas (Ferrada, 2008).

OE3: Constatar si existen diferencias significativas en el efecto de las variables que explican la conmutación entre Centros Nodales

Se trata de evaluar si el impacto de los coeficientes asociados a cada variable es significativamente distinto entre los centros nodales, para ello se modelará una ecuación por Centro.

Se debe precisar que entre aquellas zonas que se obtenga correlación significativa entre perturbaciones de distintas ecuaciones (según el procedimiento descrito en el punto anterior), el estimador que se calcula en cada ecuación por separado no es el más eficiente, ni siquiera asintóticamente. Además, en este caso, se estimarían incorrectamente las varianzas de los estimadores y se usarían indebidamente en la inferencia. Así pues, la forma adecuada de estimar el conjunto de ecuaciones que presenten correlación por medio de los errores es mediante un sistema de ecuaciones simultáneas, desde estas obtenemos el mejor estimador posible. Concretamente, se modeliza mediante un sistema de ecuaciones relacionada mediante los errores, SUR.

En el caso de un SUR el modelo econométrico se especifica mediante M ecuaciones, que se puede generalizar de la siguiente forma:

$$
\text { lnconmuta }_{m}=X_{m} \beta_{m}+U_{m}
$$

Siendo $m=1,2, \ldots M$, siendo $M$ el número de ecuaciones, $l_{-}$conmuta ${ }_{m}$ es un vector de dimensión $N^{*} 1 ; X_{m}$, es una matriz $N^{*} K_{m} ; \beta_{m}$, un vector $K_{m}{ }^{*} 1$ y $U_{m}$, un 
vector $N^{*} 1$, siendo la matriz $X$ diagonal en bloques donde cada bloque es la matriz $X_{m}$ correspondiente a cada ecuación (Novales, 1996).

Los supuestos del modelo SUR establecen que no existe autocorrelación ni heterocedasticidad dentro de las ecuaciones, pero existe correlación entre las perturbaciones de distintas ecuaciones. Entonces la matriz de varianzas y covarianzas de cada ecuación es de la forma: $E\left[U_{m} U_{m}^{\prime}\right]=\sigma_{m}^{2} I_{N} \quad m=1,2, \ldots, M$; donde $I_{N}$ es la matriz identidad de orden $N$. Además las perturbaciones de la matriz de varianzas y covarianzas tienen media nula. De este modo, sucede que en cada ecuación la matriz de varianzas y covarianzas es el producto de un escalar por una matriz identidad.

Siguiendo a Novales (1996, pág. 278) y Greene (1999, pág. 44) el procedimiento de estimación de un modelo SUR, cuando son conocidos los elementos de la matriz $\Omega$, es el de MCG, en este caso es posible obtener un estimador consistente, asintóticamente normal y el más eficiente asintóticamente.

Por tanto, se realizan dos tipos de modelación, dependiendo si las perturbaciones asociadas a las ecuaciones de distintas zonas están o no correlacionadas; para el primer caso se estimará por medio de un SUR; al contrario, si no hay correlación se modela cada ecuación por separado. No obstante, sea cual sea la forma de estimar, para verificar la cuarta hipótesis se utilizará el test de Wald.

\section{RESULTADOS}

Conforme con la metodología expuesta anteriormente y siguiendo el mismo orden se exponen los resultados, esto es, en primer lugar se estima el impacto de ciertas variables en la conmutación intrapatagónica, para ello se realizan las estimaciones uniecuacionales por MCG incluyendo una dummy por Centro Nodal; luego se evalúa si hay otros factores que sean comunes para explicar la conmutación entre zonas, en este caso se verifica la existencia de correlación entre las perturbaciones de distintas ecuaciones, una por cada Centro Nodal; finalmente, se constata si hay diferencias entre los determinantes de la conmutación, para ello se estima una ecuación por Centro Nodal o mediante un SUR según corresponda, de modo de aplicar contrastes de igualdad de coeficiente entre las ecuaciones.

\section{Efecto de los determinantes de la conmutación}

El modelo uniecuacional se aprecia en la Tabla 3. Como se observa, en general en ambos modelos los efectos son consistentes. A continuación se describe el impacto de cada variable, para ello tendremos en cuenta que muchas de ellas están expresadas en términos logarítmicos, inclusive la dependiente.

Se obtiene que claramente un aumento en el costo de transporte reduce la proporción de conmutantes hacia los Centros Nodales de la Patagonia, lo que sin duda es de preocupación, pues en gran parte del territorio el costo de transporte se eleva por el 
alto precio del petróleo entre la región de Valdivia y Coyhaique. Esta información es de interés para las políticas públicas, porque la reducción de estos costos puede tener alto impacto en el mercado laboral.

TABLA 3

ESTIMACIONES DE MCG; VARIABLE DEPENDIENTE LNCONMUTA Y VARIABLE DE PONDERACION F_TRABAJO_MUJER

\begin{tabular}{|c|c|c|c|c|}
\hline & \multicolumn{2}{|c|}{ Modelo 1} & \multicolumn{2}{|c|}{ Modelo 2} \\
\hline & Coeficiente & Desv. Típica & Coeficiente & Desv. Típica \\
\hline Const & $18,7945^{*}$ & 11,1266 & $18,0592 *$ & 10,7254 \\
\hline lnCosto & $-1,2220 * * *$ & 0,3656 & $-1,7946 * * *$ & 0,3672 \\
\hline lnDistancia & $-3,2408^{*}$ & 1,6891 & $-4,8442 * * *$ & 1,6196 \\
\hline lnDistancia ${ }^{2}$ & $0,2679 * *$ & 0,1250 & $0,4169^{* * *}$ & 0,1245 \\
\hline Inpoblac & $-0,0238^{* * * *}$ & 0,0090 & $-0,0177^{*}$ & 0,0090 \\
\hline Ipob* $^{*}$ dist $^{-1}$ & $5,6444 * * *$ & 2,0863 & 1,7820 & 2,0866 \\
\hline Educa_superior & $-4,3490$ & 2,7923 & $-7,9364 * * *$ & 2,7224 \\
\hline Calidad_empleo & $-5,5025^{*}$ & 2,9827 & $-4,7536^{*}$ & 2,7800 \\
\hline F trabajo_mujer & $-18,7983^{* *}$ & 8,0912 & $-16,5997 * *$ & 7,6255 \\
\hline Inactividad & $-10,4418 * *$ & 4,8422 & $-12,3533 * * *$ & 4,4968 \\
\hline Edad3 (>= 45 años) & $-8,1439 * *$ & 3,8414 & $-7,0335^{*}$ & 3,5900 \\
\hline lning_monetario & 1,2617 & 0,8116 & $2,0116^{* * * *}$ & 0,7681 \\
\hline Osorno & & & $-0,0511$ & 0,3645 \\
\hline Puerto Montt & & & $1,4277 * * *$ & 0,3732 \\
\hline Coyhaique & & & $2,3009 * * *$ & 0,4264 \\
\hline \multirow[t]{2}{*}{ Punta Arenas } & & & $1,3146^{* * *}$ & 0,4463 \\
\hline & $\mathrm{R} 2=0,5598$ & Akaike $=744,11$ & $\mathrm{R} 2=0,6305$ & Akaike $=705,84$ \\
\hline$N=264$ & $\mathrm{R} 2$ ajustado $=0,5461$ & Valor $p(F)=0,000$ & R2 ajustado $=0,6082$ & Valor $p(F)=0,00$ \\
\hline
\end{tabular}

Fuente: Elaboración propia.

***significación al 1\%; **significación al 5\%; *significación al $10 \%$.

Otro efecto negativo y con mayor intensidad se aprecia en la distancia, esto quiere decir que para la población que reside en lugares más distantes a los Centros Nodales su posibilidad de conmutar es menor; no obstante, también se observa que el resultado de la misma variable elevada al cuadrado es positiva, lo que nos indica que existe una característica clave: esto es, que en territorios tanto cercanos como muy distantes el efecto es similar, dejando como más recurrente las distancias medias. 
Por otro lado, un mayor peso demográfico en el Centro Nodal respecto del territorio de residencia tiene un efecto negativo en la variación de la proporción de conmutantes hacia ese Centro, lo que no es coherente con la idea de que la concentración de población en determinadas ciudades pueden incidir en la decisión de los trabajadores a laborar en un territorio distinto del lugar de residencia. Sin embargo, en general en la Patagonia los territorios menos poblados se encuentran muy lejanos de los Centros Nodales, por tanto el efecto del índice poblacional pudiese estar afectado por la distancia al Centro. Efectivamente, al revisar la variable que controla conjuntamente población con el inverso de la distancia se obtiene un efecto positivo; lo anterior es muy interesante, pues el resultado nos dice que variaciones positivas en el tamaño poblacional de territorios de origen cercanos tienen efectos claramente positivos en la variación de la proporción de trabajadores que conmuten así, en este caso, el efecto del peso de la población actúa como una fuerza de atracción de conmutantes, y a distancias mayores pierde su efecto.

A su vez, claramente la variación en la proporción de personas que conmutan a los Centros Nodales con motivos laborales disminuye en la medida que en los territorios de residencia se tenga mayor nivel educativo, calidad de trabajo, actividad laboral y exista una mayor proporción de fuerza de trabajo femenina o se tenga mayor proporción de adultos; sin embargo las variaciones positivas en el ingreso monetario del hogar motivan la movilidad.

Para el modelo 2, cuyos resultados están en la Tabla 3, se tiene como referencia el Centro Nodal de la comuna de Valdivia; se aprecia que, con excepción de Osorno, en todos los otros Centros Nodales su presencia genera efecto significativo y positivo. Sin embargo, estas estimaciones aún no nos permiten evaluar diferencias en el impacto de las variables entre Centros, para verificarlo en primer lugar se analiza si hay factores comunes entre los Centros Nodales que expliquen el comportamiento de la conmutación y que estén ocultas en las perturbaciones, lo que se revisa en el siguiente punto.

\section{Factores comunes que expliquen la conmutación entre Centros Nodales de la Patagonia}

Como se ha explicado, en la Patagonia chilena se podrían observar ciertas características comunes, las que quedarían recogidas en las perturbaciones si se estimara una ecuación por Centro Nodal. En la Tabla 4 se observa que la correlación entre las variables contenidas en los vectores de error de las ecuaciones que representan la conmutación hacia los Centros Nodales de Puerto Montt y Valdivia, y Puerto Montt y Osorno, son significativas; no obstante, la relación entre los otros Centros no es significativamente distinta de cero, lo que marca una diferencia importante en la conmutación hacia los Centros del norte y del sur de la Patagonia. 
TABLA 4

CORRELACION ENTRE RESIDUOS DE ECUACIONES

PARA CADA CENTRO NODAL

\begin{tabular}{|l|c|c|c|c|}
\cline { 2 - 5 } \multicolumn{1}{c|}{} & U Osorno & U P. Montt & U Coyhaique & U P. Arenas \\
\hline U Valdivia & $-0,103$ & $0,4775^{* * *}$ & $-0,0358$ & 0,1264 \\
U Osorno & & $-0,2216^{*}$ & 0,2059 & $-0,039$ \\
U P. Montt & & & $-0,2010$ & 0,1538 \\
U Coyhaique & & & & $-0,0392$ \\
\hline
\end{tabular}

Fuente: Elaboración propia.

****ignificación al 1\%; *significación al $10 \%$.

Por tanto, se verifica que la conmutación hacia Centros localizados en la parte norte de la Patagonia están siendo impactados por variables que no conocemos (y se encuentran en las perturbaciones) y que son comunes entre ellas, lo que no ocurre en el caso de la zona sur.

Por otro lado, se sospecha la existencia de variables explicativas comunes en espacios geográficos que agrupan a varios Centros Nodales. Para ello, a partir de la matriz de correlaciones de las perturbaciones (Tabla 4), se calcula el test de multiplicadores de Lagrange para tres grupos: los residuos asociados a todas las ecuaciones que explican la conmutación a los Centros Nodales de la Patagonia; los errores de las estimaciones asociadas a Valdivia, Osorno y Puerto Montt, y los de Puerto Montt, Coyhaique y Punta Arenas, resultados que se aprecian en la Tabla 5.

\section{TABLA 5}

TEST DE LAGRANGE, INCORRELACION ENTRE PERTURBACIONES DE DISTINTAS ECUACIONES

\begin{tabular}{|l|c|c|c|}
\hline \multicolumn{1}{|c|}{ Variables de la matriz de correlaciones } & $\lambda_{L M}$ & $\chi_{g l ; 0,01}^{2}$ & $\begin{array}{c}\mathrm{H}_{0}=\text { matriz de } \\
\text { correlaciones } \\
\text { diagonal }\end{array}$ \\
\hline UValdivia, UOsorno, UP_Montt, & 19,89 & 23,$21 ; \mathrm{gl}=10$ & No se rechazan \\
U Coyhaique, UP_Arenas & 13,81 & 11,$34 ; \mathrm{gl}=3$ & Rechaza \\
UValdivia, UOsorno, UP_Montt & 3,148 & 11,$34 ; \mathrm{gl}=3$ & No se rechazan \\
UP_Montt, UCoyhaique, UP_Arenas & & \\
\hline
\end{tabular}

Fuente: Elaboración propia. 
De la Tabla 4 se obtuvo que no hay correlación entre los errores de las estimaciones asociadas a Coyhaique y Punta Arenas. En la Tabla 5 se agrega el Centro Nodal de Puerto Montt, al respecto los resultados son concluyentes, no existe correlación entre las perturbaciones de las distintas ecuaciones que representan la conmutación hacia los Centros ubicados en el sur de la Patagonia, es decir, los vectores son independientes, por tanto no se observan factores asociados entre ellos.

Por otro lado, la hipótesis para los errores de las ecuaciones que modelan la conmutación hacia los Centros Nodales del norte (Valdivia, Osorno y Puerto Montt) se rechaza; si bien en la evaluación de la Tabla 4 no se observó correlación entre Osorno y Valdivia, claramente si se considera toda la zona norte de la Patagonia se concluye que existe información común que explica la conmutación a sus Centros Nodales y que se encuentra en las perturbaciones.

Luego, los resultados permiten corroborar lo señalado en la introducción respecto de la existencia de evidencia de comportamientos distintos entre la zona norte y sur de la Patagonia, de tal manera que hay variables asociadas en la conmutación asociada hacia los Centros Nodales de la zona norte, posiblemente por estar más cercanos espacialmente por características culturales o económicas.

\section{Diferencias en el efecto de los factores que explican la conmutación de cada Centro Nodal}

El propósito aquí es evaluar si el efecto de las variables que explican la conmutación hacia los Centros Nodales impacta de forma distinta entre ellos.

Siguiendo lo planteado en la metodología, y de acuerdo con los resultados obtenidos en el punto anterior, se computan 5 ecuaciones de dos formas: (1) para explicar la conmutación hacia los Centros Nodales de Valdivia, Osorno y Puerto Montt se estima mediante un SUR, pues encontramos correlación entre los términos de perturbación de cada ecuación; (2) para modelar la conmutación hacia Coyhaique y Punta Arenas mediante MCG, una ecuación por Centro Nodal, porque como ya se ha señalado las perturbaciones en estos casos están incorrelacionadas.

Ahora bien, como se ha señalado, para probar la hipótesis en este caso, se realiza inferencia sobre los parámetros estimados, aplicando contrastes de significación conjunta para aquellos coeficientes obtenidos ya sea por medio de SUR o MCG; concretamente, mediante el test de Wald se evalúa la hipótesis de igualdad de coeficiente para los coeficientes de cada variable, los resultados se presentan en las Tablas 6 y 7.

Como se aprecia en la Tabla 6 el efecto, tanto de la distancia, el costo de transporte como el índice poblacional, para explicar la conmutación a los Centros Nodales de Valdivia, Osorno y Puerto Montt es significativamente diferente; sin embargo, las variables asociadas a características sociodemográficas y mercado laboral tienen impacto similar; ello es relevante, ya que indicaría que si se plantea dinamizar en este territorio el mercado laboral, las comunas debiesen abordar políticas individuales en el primer caso y conjuntas para el segundo. 


\section{TABLA 6}

PRUEBA DE WALD POR VARIABLE PARA LAS ESTIMACIONES DE LOS CENTROS NODALES DEL NORTE DE LA PATAGONIA

\begin{tabular}{|l|l|c|c|c|}
\hline \multicolumn{1}{|c|}{ Variable } & Hipótesis nula & $\chi^{2}$ & Pvalor & \\
\hline InDistancia & $\mathrm{C}(2)=\mathrm{C}(14)=\mathrm{C}(26)$ & 19,25203 & 0,000066 & Rechaza \\
InCosto & $\mathrm{C}(3)=\mathrm{C}(15)=\mathrm{C}(27)$ & 12,48898 & 0,001941 & Rechaza \\
Distancia2 & $\mathrm{C}(4)=\mathrm{C}(16)=\mathrm{C}(28)$ & 15,86451 & 0,000359 & Rechaza \\
\hline InIpoblación & $\mathrm{C}(5)=\mathrm{C}(17)=\mathrm{C}(29)$ & 1,759452 & 0,414897 & No se rechazan \\
\hline Ipob*dist $^{-1}$ & $\mathrm{C}(6)=\mathrm{C}(18)=\mathrm{C}(30)$ & 6,807586 & 0,033247 & Rechaza \\
Educa_superior & $\mathrm{C}(7)=\mathrm{C}(19)=\mathrm{C}(31)$ & 2,076990 & 0,353987 & No se rechazan \\
Calidad_empleo & $\mathrm{C}(8)=\mathrm{C}(20)=\mathrm{C}(32)$ & 2,979602 & 0,225417 & No se rechazan \\
F de trabajo_mujer & $\mathrm{C}(9)=\mathrm{C}(21)=\mathrm{C}(33)$ & 2,174204 & 0,337192 & No se rechazan \\
Inactividad & $\mathrm{C}(10)=\mathrm{C}(22)=\mathrm{C}(34)$ & 0,934894 & 0,626600 & No se rechazan \\
Edad3 (>=45 años) & $\mathrm{C}(11)=\mathrm{C}(23)=\mathrm{C}(35)$ & 3,299495 & 0,626600 & No se rechazan \\
Ining_monetario & $\mathrm{C}(12)=\mathrm{C}(24)=\mathrm{C}(36)$ & 3,707682 & 0,156634 & No se rechazan \\
\hline
\end{tabular}

Fuente: Elaboración propia.

\section{TABLA 7}

PRUEBA DE WALD POR VARIABLE PARA LAS ESTIMACIONES DE LOS CENTROS NODALES DEL SUR DE LA PATAGONIA

\begin{tabular}{|l|c|c|c|l|}
\hline \multicolumn{1}{|c|}{ Variable } & Hipótesis nula & $\chi^{2}$ & Pvalor & \\
\hline InDistancia & $\mathrm{C}(38)=\mathrm{C}(50)$ & 0,011904 & 0,913119 & No se rechazan \\
lnCosto & $\mathrm{C}(39)=\mathrm{C}(51)$ & 0,508899 & 0,475616 & No se rechazan \\
Distancia2 & $\mathrm{C}(40)=\mathrm{C}(52)$ & 0,049453 & 0,824018 & No se rechazan \\
lnIpoblación & $\mathrm{C}(41)=\mathrm{C}(53)$ & 0,700309 & 0,402680 & No se rechazan \\
Ipob*dist $^{-1}$ & $\mathrm{C}(42)=\mathrm{C}(54)$ & 2,301706 & 0,129232 & No se rechazan \\
Educa_superior & $\mathrm{C}(43)=\mathrm{C}(55)$ & 4,427239 & 0,035370 & Rechaza \\
Calidad_empleo & $\mathrm{C}(44)=\mathrm{C}(56)$ & 2,283519 & 0,130755 & No se rechazan \\
F de trabajo_mujer & $\mathrm{C}(45)=\mathrm{C}(57)$ & 4,240294 & 0,039475 & Rechaza \\
Inactividad & $\mathrm{C}(46)=\mathrm{C}(58)$ & 0,311946 & 0,576489 & No se rechazan \\
Edad3 (>=45 años) & $\mathrm{C}(47)=\mathrm{C}(59)$ & 0,560081 & 0,454228 & No se rechazan \\
Ining_monetario & $\mathrm{C}(48)=\mathrm{C}(60)$ & 6,437904 & 0,011171 & Rechaza \\
\hline
\end{tabular}

Fuente: Elaboración propia. 
En cambio en la modelación de la conmutación hacia los Centros Nodales de la zona sur de la Patagonia (Tabla 7) se verifica igualdad de los coeficientes en las variables que se había rechazado en el norte, y no se rechaza la hipótesis nula en la mayoría de los casos. Concretamente, el efecto del costo, la distancia y el indicador de población es el mismo para explicar la conmutación tanto hacia Coyhaique como a Punta Arenas. Ello se puede explicar porque las distancias a los Centros Nodales son grandes, además, hay pocos medios de transporte y son costosos.

Al contrario, se rechaza la hipótesis para las variables educación superior, fuerza de trabajo femenina e ingreso monetario, es decir, entre esos territorios existen diferencias significativas en el efecto que esas variables ejercen sobre la conmutación laboral a Coyhaique o Punta Arenas. Posiblemente, ello permitiría promover acciones de políticas públicas en estas materias de forma coordinada, porque su retorno tendría el mismo impacto.

\section{CONCLUSIONES}

En este trabajo hemos estimado el impacto de ciertas variables en las decisiones de movilidad de los trabajadores desde distintos territorios de la Patagonia chilena hacia los Centros Nodales de ella, como la distancia, los costos de transporte y la población. Además se analiza si existen factores comunes que expliquen la conmutación entre Centros Nodales y se evalúa si hay diferencias significativas en el efecto de las variables que explican la conmutación entre los Centros. Estos propósitos han requerido un despliegue metodológico que ha sido expuesto con detalle. A continuación, a partir de los resultados, se concluye sobre la base de las hipótesis inicialmente formuladas.

$H_{01}$ : La Patagonia tiene un comportamiento moderno en el sentido que el costo de traslado y la distancia entre territorios de origen y destino no impactan significativamente a la conmutación

A partir de las estimaciones de las ecuaciones 1 y 2 , se obtiene que una disminución porcentual, tanto del costo de traslado como de la distancia, impactará positivamente en la variación de la proporción de conmutantes a los Centros Nodales de la Patagonia; no obstante, cuando los trabajadores residen en zonas muy distantes el efecto es contrario, probablemente derivado de mayores alternativas de transporte de larga distancia. Por otro lado, la mayor concentración de población en Centros Nodales constituye un efecto de atracción para captar trabajadores que residen en territorios cercanos a ellos.

Con todo, se tienen aún grandes desafíos en esta materia, particularmente por las dificultades espaciales derivadas de la diversidad geográfica del territorio. Así, para residentes con distancias intermedias a los Centros Nodales sería deseable generar 
incentivos que permitan reducir los costos de traslado. Es claro que los avances tecnológicos en materia de transporte y accesibilidad aún constituyen una elevada barrera para conmutar en esta parte del país.

$H_{02}$ : Las características laborales de los territorios de residencia no impactan en conmutar hacia los Centros Nodales de la Patagonia chilena

Basados en las características laborales estudiadas en este trabajo, se obtiene que todas las variables son estadísticamente significativas; así, la variación en la proporción de conmutantes para trabajar en los Centros Nodales disminuye; si en territorios de residencia mejoran los niveles educativos y se incrementará si el índice de calidad del empleo y la proporción de fuerza de trabajo femenina aumentan. Por tanto, se aprecia que la conmutación está asociada principalmente a trabajos de menor cualificación, y ayuda a resolver las bajas tasas de participación laboral de muchos territorios que no son Centros Nodales, lo que posiblemente ocurre de forma diferenciada entre el norte y sur de la Patagonia. En el sur los costos de transporte son mayores, en consecuencia, el salario de reserva de la población económicamente activa es mayor, de esta forma, para participar en el mercado laboral exigirá un salario de mercado mucho mayor.

$H_{03}$ : La conmutación con motivos laborales desde distintos territorios de la Patagonia hacia los Centros Nodales está también impactada por factores comunes a la zona

Como se ha señalado, es posible que además de las variables incluidas en las estimaciones existan otras que estén asociadas para explicar la conmutación en el territorio, o en parte de este. Los resultados permiten corroborar ciertos patrones comunes en la zona norte de la Patagonia chilena; lo que posiblemente se relaciona tanto a aspectos geográficos como a menor distancia relativa, a un mayor peso de la población en los Centros Nodales, estructura económica y aspectos culturales, entre otros. En cambio en el sur las estructuras se plantean como independientes, derivado de las grandes distancias entre ellas, de la dispersión geográfica y de diferencias importantes en la aplicación de políticas públicas en cada caso.

$H_{04}$ : Existen diferencias significativas en el efecto de las variables conocidas para explicar la conmutación hacia los distintos Centros Nodales de la Patagonia

Finalmente, se ha probado que en el norte de la Patagonia las variables costo, distancia y población, tienen impacto significativamente distinto para explicar la conmutación a los Centros Nodales. Por el contrario, se ha encontrado que no existen diferencias en el impacto del costo de transporte para conmutar a Coyhaique o Punta 
Arenas. En este sentido, en especial en períodos en baja en el ciclo económico regional, una forma de enfrentar estas dificultades de demanda laboral en algunos territorios podría estar vincula a generar incentivos para reducir los costos de transporte; claro está que en el norte de la Patagonia debe ser como una política diferenciada y en el sur como una estrategia zonal.

Por otro lado, las variables educación, fuerza de trabajo de la mujer e ingreso monetario del hogar, tienen efecto idéntico para explicar la conmutación hacia Valdivia, Osorno y Puerto Montt y diferente en explicar la conmutación hacia Punta Arenas y Coyhaique. De esta forma, para incentivar la movilidad se requieren políticas conjuntas con relación a estas variables en el norte y diferenciadas en el sur.

Este trabajo ofrece un conjunto de argumentos que permiten abrir un espacio de discusión para proponer políticas diferenciadas territorialmente, con el objetivo de mejorar oportunidades laborales a los residentes de la Patagonia chilena.

A su vez, indagar en el fortalecimiento de estos territorios mediante enfoques de desarrollo diferenciados, aplicando políticas públicas que permitan, por ejemplo, una oferta competitiva de servicios, esto es, haciendo que los trabajadores conmuten al Centro Nodal (centro productivo) pero demanden servicios en la zona de residencia, y mantengan las relaciones significativas del lugar de residencia. Un aspecto importante aquí es que conmutar, y por lo tanto no migrar, permite sostener otro tipo de relaciones en el territorio. No obstante, estos desafíos requieren de decisiones políticas que además mitiguen los impactos de una mayor oferta en los Centros, y en definitiva que den sostenibilidad al territorio en su conjunto.

Finalmente, el diseño metodológico aquí planteado constituye una contribución, pues no solo ha permitido resolver todos los desafíos inicialmente plateados, sino que puede extrapolarse a otros espacios territoriales.

\section{BIBLIOGRAFIA}

ABALOS, M. y D. PAREDES (2016). Una metodología para delimitar regiones urbanas funcionales $(R U F)$ usando la conmutación a larga distancia: Evidencia empírica para Chile. Antofagasta, Chile: Universidad Católica del Norte.

AROCA, P. y M. ATIENZA (2008). "La conmutación regional en Chile y su impacto en la Región de Antofagasta”, EURE, 102, pp. 97-120.

ASCENCIO, M. y O. PINO (2015). "La conmutación laboral femenina en la Región del Biobío: un análisis exploratorio desde la perspectiva de género", Horizontes Empresariales , 14 (1), pp. 20-29.

FERRADA, L. M. (2008). Comparaciones regionales en cuanto a participación laboral de la Mujer. Valladolid, España: Universidad de Valladolid.

FERRADA, L. M.; SOZA-AMIGO, S. y MANCILLA, C. (2007). “¿Son los Centros Nodales de la Patagonia Chile espacios funcionales al territorio? Un análisis desde la movilidad laboral". (En revisión).

FUJITA, M. y P. KRUGMAN (2004). "La nueva geografía económica: pasado, presente y futuro", Investigaciones Regionales, primavera (4), pp. 177-206.

GREENE, W. (1999). Análisis Econométrico .Tercera ed. Madrid, España: Prentice Hall.

INE (2016). Presentación Censo 2017. Recuperado en junio de 2017, de http://www.censo2017.cl/wpcontent/uploads/2016/12/pc2016_region-comuna-13122016.pdf 
JAMETT, I. y D. PAREDES (2013). “Conmutación de larga distancia en Chile: Estimando el premio por trabajar muy lejos de casa", Estudios de Economía, 40 (2), pp. 179-209.

MANCILLA, C. y S. SOZA-AMIGO (2016). Conmutación intra e interregional, sectores económicos y niveles. 8vo Encuentro de la Sociedad Chilena de Estudios Regionales. Santiago: Mimeo (SOCHER, 2016).

NOVALES, A. (1996). Econometria. Segunda ed. Madrid: McGraw-Hill.

SAID, J. (2012). Patagonia. Buenos Aires: Sudamericana.

SOZA-AMIGO, S. (2016). "Conmutación centrípeta-centrífuga: ¿es la vocación productiva una motivación para conmutar?", IDESIA , 34 (1), pp. 55-67.

SOZA-AMIGO, S.; C. ROSALES y P. AROCA (2016). "Análisis de los centros primados vinculados al turismo en el Parque Nacional Puyehue". Revista de Geografía Norte Grande, 65, pp. 179-195. 


\section{ANEXO}

PATAGONIA CHILENA Y COMUNAS NODALES PRIMADAS

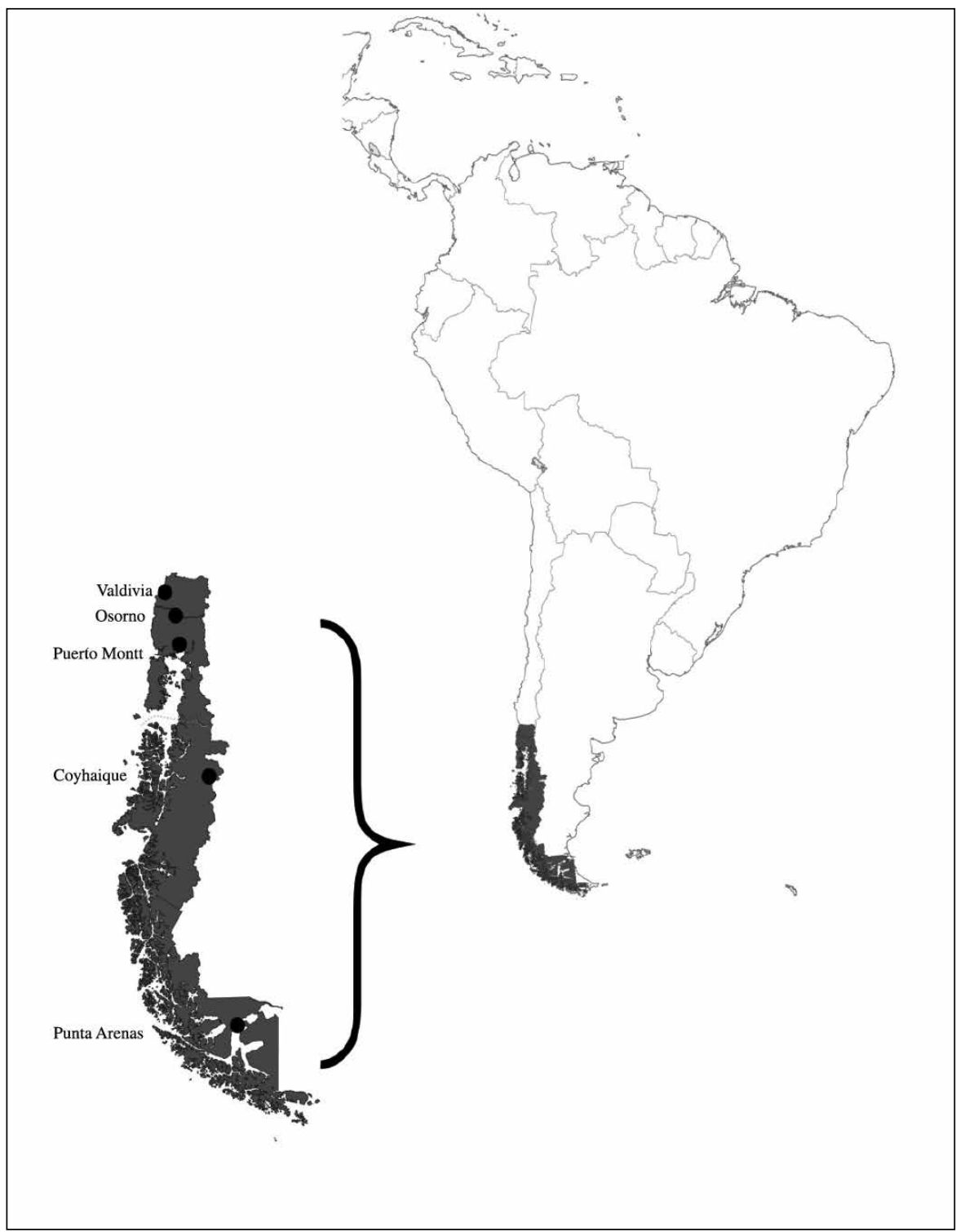

Fuente: Ferrada, Soza-Amigo y Mancilla, 2017. 